\title{
O IMPACTO DO ENVOLVIMENTO PARENTAL NO DESEMPENHO ACADÊMICO DE CRIANÇAS ESCOLARES ${ }^{1}$
}

\author{
Fabiana Cia* \\ Renata Christian de Oliveira Pamplin\# \\ Lúcia Cavalcanti de Albuquerque Williams ${ }^{\pi}$
}

\begin{abstract}
RESUMO. O objetivo deste trabalho foi estudar a relação entre o envolvimento dos pais na educação dos filhos e o desempenho acadêmico destes. Participaram deste estudo 110 crianças da $4^{\text {a }}$ série do Ensino Fundamental. As crianças preencheram o questionário "Qualidade da interação familiar na visão dos filhos" e foram avaliadas usando-se o Teste de Desempenho Escolar - TDE. Observou-se que quanto maior a freqüência de comunicações entre ambos os pais e seus filhos e a participação de ambos os pais nas atividades escolares, culturais e de lazer dos filhos, melhor o desempenho acadêmico das crianças. Tais resultados sugerem a importância do envolvimento parental no desempenho acadêmico dos filhos e, em caso de déficits nessa área, a necessidade de programas, visando melhorar a qualidade das interações entre pais e filhos.
\end{abstract}

Palavras-chave: envolvimento parental, desempenho acadêmico, crianças escolares.

\section{THE IMPACT OF PARENTAL INVOLVEMENT ON CHILDREN'S ACADEMIC PERFORMANCE}

\begin{abstract}
The relationship between parental involvement in children's education and children's academic performance is provided. A total of 110 (fourth grade children) participated in this study. The children completed the questionnaire "Quality of family interaction according to the children" and were evaluated by the using the School Performance Test (TDE). Results showed that the higher the frequency of mother-and father-child communication and the greater the parents' involvement in the children's school, cultural and leisure activities, the higher the children's academic performance. Results suggest the importance of parental involvement on the children's academic performance. In case of deficits is this area, the urgent need of programs aiming at improving the quality of the interactions between parents and their children, is enhanced.
\end{abstract}

Key words: Parental involvement, academic performance, children.

\section{EL IMPACTO DEL ENVOLVIMIENTO PARENTAL EN EL DESEMPEÑO ACADÉMICO DE NIÑOS ESCOLARES}

RESUMEN. El objetivo de este trabajo fue estudiar la relación entre el envolvimiento de los padres en la educación de los hijos y el desempeño académico de estos. Participaron de este estudio 110 niños de la $4^{\mathrm{a}}$ serie de la Enseñanza Fundamental. Los niños rellenaron el cuestionario "Cualidad de la interacción familiar en la visión de los hijos" y fueron evaluados usándose el Teste de Desempeño Escolar - TDE. Se observó que cuanto mayor la frecuencia de comunicaciones entre ambos los padres y sus hijos y la participación de ambos los padres en las actividades escolares, culturales y de lazer de los hijos, mejor el desempeño académico de los niños. Tales resultados sugieren la importancia del envolvimiento parental en el desempeño académico de los hijos y, en caso de déficits en esa área, la necesidad de programas, visando mejorar la cualidad de las interacciones entre padres e hijos.

Palabras-clave: Envolvimiento parental, desempeño académico, niños escolares.

\footnotetext{
Apoio: Fapesp.

Psicóloga. Doutoranda do Programa de Pós-Graduação em Educação Especial Universidade Federal de São Carlos-UFSCar.

* Pedagoga. Doutoranda do Programa de Pós-Graduação em Educação Especial Universidade Federal de São Carlos-UFSCar.

^ Doutora. Professora Titular do Programa de Pós-Graduação em Educação Especial e do Departamento de Psicologia da Universidade Federal de São Carlos-UFSCar.
} 
Tanto no contexto brasileiro quanto no norteamericano, a maioria das crianças classificadas e rotuladas como portadoras de necessidades educacionais especiais não tem uma excepcionalidade óbvia (Brasil, 2002; Gargiulo, 2003; Hallahan \& Kauffman, 2005), embora geralmente esses alunos apresentem dificuldades de aprendizagem ou histórico de repetência e de fracasso escolar (Pamplin, 2005). A literatura aponta vários fatores de risco para o baixo desempenho acadêmico, como o baixo nível socioeconômico na família (Ackerman, Brown \& Izard, 2004; Marturano, 2004), a exposição da criança à violência (Brancalhone, Fogo \& Williams, 2004) ou uma baixa qualidade de interação com os pais (Flouri \& Buchanan, 2003).

De fato, os pais estabelecerem um ambiente familiar acolhedor e organizarem contextos favoráveis para o desenvolvimento da criança é tido como fator de proteção diante de eventos ameaçadores a que usualmente as crianças estão expostas (Del Prette \& Del Prette, 2005; Dessen \& Costa, 2005). Em contrapartida, a exposição da criança a práticas parentais inadequadas (conflitos, violência, coerção) ou a baixo envolvimento com o pai ou com a mãe se constituem em fatores de risco para o desenvolvimento infantil, aumentando a vulnerabilidade a eventos ameaçadores, externos ao ambiente familiar, como práticas delinqüentes e envolvimento com drogas (Gomide, 2003; MacDowell \& Parke, 2005). Partindo destes pressupostos, este estudo visa estabelecer relações entre o envolvimento parental e o desempenho acadêmico dos filhos, com o objetivo de identificar possíveis fatores de risco para o baixo desempenho acadêmico de crianças escolares.

\section{A IMPORTÂNCIA DO ENVOLVIMENTO PARENTAL PARA O DESEMPENHO ACADÊMICO DOS FILHOS}

É crescente o interesse das escolas pelas pesquisas sobre envolvimento parental, pois o tema está diretamente correlacionado com o maior desempenho acadêmico dos filhos (D'Avila-Bacarji, Marturano \& Elias, 2005; Hill et al., 2004). Por exemplo, Englund, Luckner, Whaley e Egeland (2004) realizaram um estudo longitudinal com 187 crianças e suas mães, sendo tais crianças acompanhadas do nascimento até a $3^{\text {a }}$ série escolar, para verificar a influência do envolvimento, da expectativa e da qualidade da assistência parental no desempenho acadêmico dos filhos. Os resultados mostraram que a qualidade da assistência parental, quando a criança tinha três anos, estava positivamente correlacionada ao QI da criança aos cinco anos e indiretamente correlacionada ao desempenho acadêmico da criança na $1^{\mathrm{a}}$ e na $3^{\mathrm{a}}$ séries. A expectativa e o envolvimento parental no desempenho da criança na $1^{\text {a }}$ e na $3^{\text {a }}$ séries estavam diretamente correlacionados ao desempenho acadêmico dos filhos.

Cia, D’Affonseca e Barham (2004) estudaram o impacto da freqüência de envolvimento paterno sobre o desempenho acadêmico de crianças escolares. Participaram desse estudo 58 pais (homens) e seus filhos da $5^{\mathrm{a}}$ ou $6^{\mathrm{a}}$ séries. Observou-se que, quanto maior a freqüência de comunicações entre pai e filho (segundo o pai e o filho) e quanto maior o envolvimento dos pais nas atividades escolares, culturais e de lazer do filho (segundo o pai), melhor o desempenho das crianças em escrita e leitura.

Em outro estudo, realizado com famílias brasileiras, Ferreira e Marturano (2002) compararam o ambiente familiar em dois grupos de crianças que apresentavam desempenho acadêmico pobre, associado $(N=37)$ ou não $(N=30)$ com problemas de comportamento. Os resultados mostraram que o grupo de crianças com dificuldades de aprendizagem e com problemas de comportamento, quando comparado com o das crianças que apresentavam dificuldades de aprendizagem mas não tinham problemas de comportamento, apresentou menos recursos e maior adversidade, incluindo: envolvimento e suporte dos pais (passeios, atividades compartilhadas com os pais no lar, oferta de brinquedos e outros materiais promotores de desenvolvimento, pessoas a quem a criança recorre para pedir ajuda ou conselho); menor nível socioeconômico, menos recursos no ambiente familiar, maior número de eventos adversos (adversidade nas relações parentais, eventos adversos na vida pessoal e escolar e problemas nas relações interpessoais); adversidades do ambiente familiar (práticas educativas inadequadas, problemas no relacionamento pais-filhos).

Em uma revisão de literatura, Hong e Ho (2005) selecionaram estudos longitudinais, avaliando os participantes nos anos de 1988, 1990 e 1992. Após esta seleção, foi usada uma amostra randômica de 6000 estudantes. O objetivo desse estudo foi verificar a influência do envolvimento parental (comunicação entre pais e filhos, aspiração educacional dos pais, participação parental e supervisão parental), do autoconceito, do locus de controle e da aspiração educacional dos estudantes sobre o desempenho acadêmico de crianças de quatro grupos étnicos que vivem nos Estados Unidos. Pôde-se verificar que a comunicação entre pais e filhos influenciou diretamente o desempenho acadêmico das crianças 
hispânicas e brancas e teve um efeito direto no crescimento acadêmico em crianças asiáticas e brancas. A aspiração educacional parental influenciou diretamente o desempenho acadêmico de crianças asiáticas, afro-americanas e brancas e influenciou diretamente o crescimento acadêmico das crianças asiáticas e brancas. A supervisão parental apresentou influência direta no crescimento acadêmico de crianças afro-americanas. Além disso, de modo geral, o envolvimento parental estava positivamente correlacionado com o locus de controle, o autoconceito e a aspiração educacional dos estudantes, que, por sua vez, estavam indireta e positivamente correlacionados ao desempenho e crescimento acadêmico das crianças.

Além da importância do envolvimento parental para o desempenho acadêmico das crianças, estudos têm evidenciado a importância da participação de ambos os pais na educação dos filhos (Caballo \& Simón, 2005; Dessen \& Costa, 2005; Duch, 2005; Overstreet, Devine, Bevans \& Efreom, 2005). A partir dos anos de 1950 houve um aumento do número de mulheres no mercado de trabalho, o que acarretou mudanças na rotina familiar e nos cuidados com os filhos (Bertolini, 2002; Cabrera, Tamis-LeMonda, Bradley, Hofferty \& Lamb, 2000; Ozgun \& Honig, 2005). Diante disso, os homens estão aumentando o tempo que despendem nos cuidados com os filhos e, conseqüentemente, tendo um importante papel na maximização do desenvolvimento infantil (Flouri, 2004; Ross, Stein, Trabasso, Woody \& Ross, 2005). Por isso as investigações sobre práticas parentais deveriam focalizar não somente a atenção da mãe, mas também a do pai e a divisão dos papéis parentais em sua influência no desenvolvimento dos filhos (Cia, Williams \& Aiello, 2005; Oliveira et al., 2002; Weber, Prado, Viezzer \& Brandenburg, 2004).

Considerando a importância do envolvimento parental para o desempenho acadêmico do filho e a escassez de estudos focalizando especificamente a participação de cada cônjuge na sua educação, este estudo teve por objetivo principal verificar a relação entre o envolvimento de ambos os pais na educação dos filhos e o desempenho acadêmico destes.

\section{MÉTODO}

\section{Participantes}

Participaram deste estudo 110 crianças da $4^{\text {a }}$ série do Ensino Fundamental, com média de idade de 10 anos, variando entre nove e 12 anos. A maioria das crianças vivia com ambos os pais $(92,7 \%)$ e exatamente a metade delas era do sexo feminino e metade do sexo masculino. Em relação ao nível socioeconômico, $0,9 \%$ era de classe socioeconômica A1; 7,3\% eram de classe socioeconômica A2; 24,5\% eram de classe socioeconômica $\mathrm{B} 1 ; 33,6 \%$ eram de classe socioeconômica B2; 30,0\% eram de classe socioeconômica $\mathrm{C}$ e $3,6 \%$ eram de classe socioeconômica D (Critério Brasil, 2006).

\section{Local da coleta de dados}

A coleta de dados ocorreu em sala isenta de ruídos, de uma escola gratuita (mantida por indústrias) localizada em uma cidade no Interior do Estado de São Paulo.

\section{Medidas avaliativas das crianças}

Para avaliar as crianças foram utilizados 0 Questionário da qualidade da interação familiar na visão dos filhos (Cia et al., 2004) e o Teste de Desempenho Escolar - TDE (Steine,1994).

O Questionário se compõe de três escalas tipo Likert, que contemplam uma diversidade de habilidades sociais educativas: (1) escala de interação entre pais e filhos ${ }^{2}(\alpha=0,82, \alpha=0,81$, para a interação pai-filho e mãe-filho, respectivamente); (2) escala de interação entre filhos e pais $(\alpha=0,82, \alpha=0,77$, para a interação pai-filho e mãe-filho, respectivamente; e (3) escala de participação dos pais nas atividades escolares, culturais e de lazer dos filhos $(\alpha=0,91$, $\alpha=0,87$, para a participação do pai e da mãe, respectivamente.

O Teste de Desempenho Escolar - TDE (Stein, 1994) foi usado para obter o índice do desempenho escolar das crianças. É um instrumento com propriedades psicométricas adequadas no que diz respeito a sua confiabilidade interna, que avalia as capacidades fundamentais para o desempenho escolar. Este teste foi concebido para a avaliação de escolares de $1^{\mathrm{a}}$ a $6^{\mathrm{a}}$ séries do Ensino Fundamental e é composto por três subtestes: escrita, aritmética e leitura.

\section{Procedimento de coleta de dados}

Antes de iniciar a coleta de dados com as crianças, foi encaminhado o Termo de Consentimento

\footnotetext{
$\mathrm{Na}$ escala de interação entre pai e filho, avalia-se a freqüência com que os pais se direcionam ao filho para se comunicarem, realizar alguma atividade, entre outros aspectos ; e na escala de interação entre filho e pai, avaliase a freqüência com que os filhos se direcionam aos pais, nessas mesmas habilidades.
} 
Livre e Esclarecido para os pais autorizarem a participação de seu filho na pesquisa. As crianças cujos pais autorizaram sua participação preencheram o questionário "Qualidade da Interação Familiar na Visão dos Filhos", em que as pesquisadoras falavam em voz alta cada item e os alunos preenchiam a freqüência que condizia com sua realidade. A aplicação deste questionário foi coletiva (em média, com 35 alunos) e teve duração média de 40 minutos. Em seguida, foi aplicado o TDE (aplicação individual). A aplicação do teste levou cerca de 60 minutos para cada aluno.

\section{Procedimento de análise de dados}

Os dados quantitativos, obtidos por meio do questionário "Qualidade da Interação Familiar na Visão dos Filhos", foram analisados com métodos descritivos, como medidas de tendência central e dispersão. Para verificar a fidedignidade das medidas no contexto deste estudo, foi realizada uma análise de consistência interna (Alpha de Cronbach) da escala como um todo (Cozby, 2002). As pontuações dos dados obtidos no TDE foram realizadas com base nos procedimentos apresentados no respectivo manual. Para comparar os dados de mães e pais foi utilizado o teste-t de Student para amostras independentes $(p<0,05)$. Para investigar a relação entre o desempenho acadêmico das crianças e o tipo e grau de envolvimento dos respectivos pais/mães, foi utilizado o teste de correlação de Pearson - aplicativo SPSS 10.0. Inicialmente as correlações foram feitas considerando as escalas de envolvimento parental como um todo; ou seja, após ter uma média de freqüência de interação dos pais e das mães com os filhos, considerando todos os itens que compõem cada uma das três escalas, fazem-se as correlações com o desempenho acadêmico das crianças. Caso não houvesse correlações com a escala, como um todo, eram feitas correlações do desempenho acadêmico com cada item das escalas separadamente.

\section{RESULTADOS}

\section{Interação entre pais e filhos}

A Tabela 1 compara a freqüência de interação entre mãe/pais e seus filhos.

Tabela 1. Interação dos Pais com os Filhos: Comparação entre Mães e Pais

\begin{tabular}{|c|c|c|c|c|c|c|}
\hline \multirow[t]{2}{*}{ Item } & \multicolumn{2}{|c|}{ Mãe } & \multicolumn{2}{|c|}{ Pai } & \multicolumn{2}{|r|}{ Teste-1 } \\
\hline & Média & $D P$ & Média & $D P$ & $t$ & $g l$ \\
\hline \multicolumn{7}{|l|}{ Seu pai/Sua mãe } \\
\hline Dá carinho a você? & 310,1 & 113,0 & 265,8 & 146,1 & $2,49 *$ & 210 \\
\hline Mantém diálogo com você? & 305,6 & 112,6 & 256,4 & 151,7 & $2,69 * *$ & 210 \\
\hline Oferece ajuda a você, quando precisa? & 300,9 & 115,1 & 260,9 & 143,2 & $2,25^{*}$ & 210 \\
\hline Elogia você? & 294,8 & 112,9 & 251,0 & 150,3 & $2,32 *$ & 209 \\
\hline Quando promete algo a você, cumpre a promessa? & 292,0 & 128,8 & 264,8 & 146,2 & 1,44 & \\
\hline Pergunta para você sobre o que aconteceu na escola? & 281,3 & 134,8 & 217,6 & 161,4 & $3,12 * *$ & 210 \\
\hline Expressa as opiniões dele a você? & 255,9 & 156,5 & 215,0 & 170,7 & 1,81 & \\
\hline Pergunta para você sobre aspectos do seu dia-a-dia? & 255,6 & 146,4 & 210,8 & 163,1 & $2,10^{*}$ & 210 \\
\hline Expressa sentimentos positivos em relação às suas atitudes? & 254,3 & 145,9 & 208,8 & 159,6 & $2,17^{*}$ & 210 \\
\hline Pergunta para você sobre seus amigos? & 224,6 & 161,6 & 171,8 & 164,9 & $2,35^{*}$ & 208,1 \\
\hline Expressa sentimentos negativos em relação às suas atitudes? & 209,2 & 161,6 & 192,1 & 160,0 & 0,77 & \\
\hline Impõe limites a você? & 206,8 & 166,6 & 208,8 & 164,9 & 0,09 & \\
\hline
\end{tabular}

Nota: A frequiência foi apontada usando uma escala que variou entre 0 , 'nunca' 12 , 'uma vez por mês' 52 , 'uma vez por semana' 104 , 'duas ou três vezes por semana' e 365 , 'todo dia'. $* p<0,05 ; * *<<0,01$.

Segundo relatos das crianças, as mães interagiam com elas com uma freqüência significativamente maior do que os pais, na maioria dos itens avaliados nesta escala. Além disso, as mães interagiam com uma freqüência significativamente maior com os filhos em relação a manter diálogo com o filho, dar carinho ao filho, oferecer ajuda ao filho, quando precisa. Os pais interagiam com uma freqüência significativamente maior com os filhos em relação a dar carinho ao filho, quando promete algo ao filho cumpre a promessa, e oferecer ajuda ao filho, quando precisa, segundo relatos das crianças.

A Tabela 2 compara a freqüência de interação entre filho e mãe/pai. 
Tabela 2. Interação dos Filhos com os Pais: Comparação entre Mães e Pais

\begin{tabular}{|c|c|c|c|c|c|c|}
\hline \multirow[t]{2}{*}{ Item } & \multicolumn{2}{|c|}{ Mãe } & \multicolumn{2}{|c|}{ Pai } & \multicolumn{2}{|r|}{$\begin{array}{c}\text { Teste } \\
-t\end{array}$} \\
\hline & Média & $D P$ & Média & $D P$ & $t$ & $g l$ \\
\hline \multicolumn{7}{|l|}{ Você } \\
\hline Dá carinho (abraços, beijos) aos seus pais? & 318,6 & 107,8 & 256,6 & 138,1 & 1,89 & \\
\hline Procura conversar com seus pais? & 308,2 & 113,2 & 268,7 & 146,5 & $2,20 *$ & 210 \\
\hline Faz elogios a seus pais? & 293,6 & 163,5 & 258,7 & 149,1 & 1,62 & \\
\hline Conta para seus pais as coisas boas ou ruins ocorridas com você em relação à escola? & 268,5 & 137,8 & 243,9 & 158,7 & $2,09 *$ & 210 \\
\hline Conta para seus pais as coisas boas ou ruins ocorridas com você em relação aos amigos? & 258,4 & 148,9 & 213,8 & 165,6 & $2,06 *$ & 210 \\
\hline Solicita que seus pais façam algo para você? & 247,8 & 151,4 & 217,5 & 162,5 & 1,40 & \\
\hline Pede para que seus pais ajudem em alguma atividade (acadêmica ou não)? & 247,7 & 151,2 & 211,1 & 162,4 & 1,70 & \\
\hline Faz perguntas para seus pais referentes ao dia a dia dele? & 232,5 & 156,4 & 192,5 & 165,9 & 1,81 & \\
\hline Expressa desejos e preferências a seus pais, dando razão para suas ações e posições? & 213,3 & 163,3 & 190,4 & 164,6 & 1,01 & \\
\hline Desafia as regras (desobedece) de seus pais? & 122,2 & 158,1 & 119,6 & 157,1 & 0,12 & \\
\hline
\end{tabular}

Nota: A freqüência foi apontada usando uma escala que variou entre 0 , 'nunca' 12 , 'uma vez por mês' 52 , 'uma vez por semana' 104, 'duas ou três vezes por semana' e 365 , 'todo dia'. $* p<0,05$.

As habilidades Você procura conversar com seus pais, Você conta para seus pais as coisas boas ou ruins ocorridas com você em relação aos amigos, Você conta para seus pais as coisas boas ou ruins ocorridas com você em relação a sua escola, foram as que as crianças apresentavam em relação às mães, com uma freqüência média significativamente maior do que em relação aos pais, segundo o relato dos filhos. Além disso, as crianças interagiam com maior freqüência com ambos os pais, em relação a dar carinho aos pais, procurar conversar com seus pais e fazer elogios aos seus pais.

\section{Participação dos pais nas atividades escolares, culturais e de lazer dos filhos}

Os dados da tabela 3 comparam a participação das mães/pais nas atividades, escolares, culturais e de lazer dos filhos.

Tabela 3. Participação dos Pais nas Atividades Escolares, Culturais e de Lazer do Filho: Comparação Entre Mães e dos Pais

\begin{tabular}{|c|c|c|c|c|c|c|}
\hline \multirow[t]{2}{*}{ Item } & \multicolumn{2}{|c|}{ Mãe } & \multicolumn{2}{|c|}{ Pai } & \multicolumn{2}{|r|}{$\begin{array}{c}\text { Test } \\
e-t\end{array}$} \\
\hline & Média & $D P$ & Média & $D P$ & $t$ & $g l$ \\
\hline \multicolumn{7}{|l|}{ Seu pai/Sua mãe } \\
\hline Acompanha o seu progresso escolar. & 310,2 & 112,7 & 259,8 & 150,4 & $2,77 * *$ & 210 \\
\hline Incentiva você a assumir responsabilidade por tarefas escolares. & 308,8 & 122,7 & 256,7 & 151,6 & $2,76^{* *}$ & 210 \\
\hline Auxilia você nas atividades de higiene (escovar os dentes, tomar banho). & 301,4 & 124,3 & 254,6 & 154,4 & $2,44 *$ & 210 \\
\hline Pede para você organizar objetos pessoais (roupas, brinquedos). & 290,0 & 132,2 & 221,0 & 163,7 & $3,39 * *$ & 210 \\
\hline Acompanha você nas refeições. & 274,9 & 145,0 & 226,2 & 162,0 & $2,31^{*}$ & 210 \\
\hline Auxilia você nas lições de casa. & 274,6 & 137,3 & 227,8 & 112,7 & $2,33^{*}$ & 210 \\
\hline Valoriza as suas conquistas esportivas. & 260,6 & 153,6 & 244,2 & 157,7 & 0,76 & \\
\hline Incentiva você a ler (livros, revistas, jornais). & 259,4 & 144,6 & 226,2 & 156,5 & 1,04 & \\
\hline Incentiva você a brincar com jogos educativos. & 259,1 & 145,2 & 218,2 & 161,7 & $1,94 *$ & 210 \\
\hline Valoriza as suas conquistas acadêmicas. & 256,7 & 153,4 & 225,6 & 161,8 & 1,17 & \\
\hline Incentiva você a realizar atividades domésticas (cuidar das próprias coisas, da casa, etc.). & 250,1 & 144,9 & 197,9 & 161,2 & $2,47 *$ & 209 \\
\hline Incentiva você a ter contato com outros adultos (tios, amigos da família, etc.). & 245,2 & 148,9 & 216,9 & 159,7 & 1,34 & \\
\hline Incentiva você a ter contato com outras crianças (leva na casa dos amigos, recebe os amigos em casa). & 245,1 & 154,4 & 202,6 & 165,3 & 1,63 & \\
\hline Assiste filme com você da sua escolha. & 240,3 & 157,1 & 201,9 & 167,9 & 1,72 & \\
\hline Passeia com você (shopping, zoológico, casa de familiares, etc.). & 234,0 & 150,9 & 207,1 & 157,6 & 1,27 & \\
\hline Brinca com você. & 205,1 & 162,9 & 186,1 & 167,7 & $1,94 *$ & 210 \\
\hline Acompanha você para se vestir. & 194,4 & 167,3 & 100,7 & 149,1 & $4,29 * * *$ & 210 \\
\hline Assiste eventos culturais com você (teatro, cinema, shows musicais). & 165,7 & 157,6 & 135,5 & 155,4 & 1,41 & \\
\hline Lê/Conta histórias para você. & 107,8 & 152,8 & 84,5 & 140,6 & 1,14 & \\
\hline
\end{tabular}

Nota: A freqüência foi apontada usando uma escala que variou entre de 0, 'nunca' 12, 'uma vez por mês' 52, 'uma vez por semana' 104, 'duas ou três vezes por semana' e 365, 'todo dia'. ${ }^{*} p<0,05 ;{ }^{* *} p<0,01 ; * * * p<0,001$.

Segundo a opinião das crianças, as mães participavam com uma freqüência média significativamente maior em nove atividades das 16 que compõem a escala, quando comparadas aos pais. De modo geral, ambos os pais realizavam com maior freqüência as atividades de: acompanhar o progresso 
escolar dos filhos, incentivar os filhos a assumir responsabilidades por tarefas escolares e auxiliar seus filhos nas atividades de higiene, de acordo com o relato das crianças.

\section{Desempenho acadêmico das crianças}

Os dados da Tabela 4 apresentam os escores médios do Teste de Desempenho Escolar obtido pelas crianças.

Tabela 4. Resultados Obtidos pelas Crianças no Teste de Desempenho Escolar

\begin{tabular}{lcccc}
\hline & Média $\boldsymbol{D P}$ & $\begin{array}{c}\text { Pontuação média para a 4 } \\
\text { série (normatização do teste) }\end{array}$ & $\begin{array}{c}\text { Pontuação } \\
\text { máxima } \\
\text { permitida }\end{array}$ \\
\hline Aritmética & 21,6 & 4,0 & $19-23$ & 38 \\
Escrita & 28,4 & 5,1 & $27-31$ & 35 \\
Leitura & 65,8 & 4,7 & $66-68$ & 70 \\
Pontuação total & 115,9 & 10,4 & $112-121$ & 143 \\
\hline
\end{tabular}

A pontuação média das crianças nos subtestes de aritmética e escrita e a pontuação total no TDE estavam na média, segundo a amostra de referência. Apenas a pontuação média em leitura estava ligeiramente abaixo da média.

\section{A relação entre o envolvimento parental e o desempenho acadêmico dos filhos}

A Tabela 5 apresenta as correlações entre os indicadores de envolvimento da mãe/pai com seus filhos e o desempenho acadêmico das crianças, segundo o TDE.

Tabela 5. Correlação Entre os Indicadores do Envolvimento de Ambos os Pais com seus Filhos e o Desempenho Acadêmico das Crianças (Teste de Pearson)

\begin{tabular}{|c|c|c|c|c|}
\hline & \multicolumn{4}{|c|}{ TDE } \\
\hline & Aritmética & Escrita & Leitura & Total \\
\hline \multicolumn{5}{|c|}{ Comunicação verbal e não verbal entre pais e filhos } \\
\hline Mãe & 0,083 & $0,251 * *$ & $0,234 *$ & $0,247 *$ \\
\hline Pai & $-0,005$ & 0,132 & $0,213 *$ & $0,196 *$ \\
\hline \multicolumn{5}{|c|}{ Comunicação verbal e não verbal entre filhos e pais } \\
\hline Mãe & 0,051 & $0,229 *$ & 0,098 & $0,278 * *$ \\
\hline Pai & $0,260 * *$ & 0,094 & $-0,014$ & 0,031 \\
\hline \multicolumn{5}{|c|}{ Participação dos pais nas atividades escolares, culturais e de lazer dos filhos } \\
\hline \multicolumn{5}{|c|}{ 然 } \\
\hline Acompanhar o seu progresso escolar. & 0,130 & $0,224 *$ & $0,188 *$ & $0,259 * *$ \\
\hline Auxilia você nas atividades de higiene (escovar os dentes, tomar banho). & $-0,041$ & $0,191 *$ & 0,025 & 0,125 \\
\hline Pede para você organizar objetos pessoais (roupas, brinquedos). & 0,006 & $0,227 *$ & 0,128 & 0,008 \\
\hline Auxilia você nas lições de casa. & 0,021 & $0,236^{*}$ & $0,220 *$ & $0,298 * *$ \\
\hline Valoriza suas conquistas acadêmicas. & 0,019 & $0,229 *$ & $-0,092$ & 0,061 \\
\hline Incentiva você a ter contato com outras crianças. & 0,056 & 0,078 & $0,291 * *$ & 0,034 \\
\hline \multicolumn{5}{|l|}{ Pai } \\
\hline Acompanhar o seu progresso escolar. & 0,080 & $0,269 * *$ & 0,012 & 0,037 \\
\hline Incentiva você a ter contato com outras crianças. & $-0,043$ & $0,216^{*}$ & $0,230 *$ & $0,244^{*}$ \\
\hline Passeia com você. & $0,214 *$ & $0,190^{*}$ & $-0,101$ & 0,048 \\
\hline Brinca com você. & $0,262 * *$ & 0,017 & 0,022 & $-0,055$ \\
\hline
\end{tabular}

Nota: ${ }^{*} p<0,05 ;{ }^{*} p<0,01$.

A escala de interação entre mães e filhos estava positivamente correlacionada com o desempenho das crianças em escrita, leitura e na pontuação total do TDE. A escala de interação entre pais (homens) e filhos estava positivamente correlacionada com o desempenho das crianças em leitura e na pontuação total do TDE. Note-se que a escala de interação entre filhos e pais estava positivamente correla-cionada com o desempenho das crianças em escrita e na pontuação total do TDE, quando se referia à interação materna, e em aritmética, quando se referia à interação paterna.
A escala de participação dos pais nas atividades escolares, culturais e de lazer não se correlacionou com o desempenho acadêmico das crianças, considerando-se a escala como um todo; no entanto, ao analisar separadamente cada atividade desenvolvida por ambos os pais que poderia se correlacionar com o desempenho das crianças, nota-se que as atividades desenvolvidas pela mãe de acompanhar o progresso escolar do filho, auxiliar o filho nas atividades de higiene, pedir para o filho organizar objetos pessoais, auxiliar o filho nas lições de casa, valorizar as conquistas acadêmicas do filho e 
incentivar o filho a ter contato com outras crianças apresentaram correlações com pelo menos um dos subtestes do TDE. As atividades desenvolvidas pelos pais que apresentaram correlações com pelo menos um dos subtestes do TDE foram as de acompanhar o progresso escolar do filho, incentivar o filho a ter contato com outras crianças, passear com o filho e brincar com o filho.

\section{DISCUSSÃO}

Segundo relatos das crianças, as mães são mais responsáveis pela sua educação e pelos seus cuidados diários do que os pais. Isto vem a demonstrar que, apesar de os pais da atualidade estarem mais envolvidos nestas atividades, quando comparados com os pais de duas décadas atrás (Cia et al., 2005; Lamb, 1997), há ainda um caminho a percorrer na direção de uma maior igualdade entre os gêneros, a fim de que haja um maior equilíbrio das responsabilidades com as mulheres (Cinamonm \& Rich, 2002; Ozgun \& Honig, 2005; Wagner, Predebon, Mosmann \& Verza, 2005).

Não obstante, segundo relatos das crianças, ambos os pais tinham um alto envolvimento na educação dos filhos, com alta freqüência de interação e de participação com eles nas atividades escolares, culturais e de lazer, o que é encorajador. No geral, esta nova divisão de tarefas, em que os cônjuges estão compenetrados da responsabilidade conjunta nas atribuições familiares, parece resultar em melhor relacionamento entre ambos e destes com os filhos (Cia, Pereira, Del Prette \& Del Prette, 2006). Além disso, essa mudança pode reverter em uma boa qualidade da interação entre os filhos, favorecendo o desenvolvimento infantil, principalmente no que diz respeito aos aspectos socioemocionais (Atzaba-Poria, Pike \& Deater-Deckard, 2004; Del Prette \& Del Prette, 2005; Scaramella \& Conger, 2004) e ao desempenho acadêmico. Paralelamente, tal prática de interação positiva é incompatível com práticas coercitivas, comprovadamente nocivas para o desenvolvimento infantil (Gomide, 2003; Brancalhone et al., 2004). De fato, neste estudo, o maior envolvimento de ambos os pais com seus filhos apresentou correlações positivas e significativas com o desempenho destes últimos em leitura, escrita e aritmética, o que vem a corroborar os resultados de outros estudos (Cia et al., 2004; D'Avila-Bacarji et al., 2005; Duch, 2005; Englund et al., 2004; Ferreira \& Marturano, 2002; Overstreet et al., 2005). Talvez isto ocorra porque, ao interagirem de modo positivo e com alta freqüência com seus filhos, tais pais têm mais oportunidades de demonstrar afeto, segurança, apoio, além de poderem motivar seus filhos nos estudos, por exemplo, demonstrando interesse pelas atividades de rotina desenvolvidas por eles.

Considerando-se as correlações entre as escalas de envolvimento parental e os subtestes do Teste de Desempenho Escolar, nota-se que há uma variedade de habilidades sociais educativas dos pais que influenciam o bom desempenho escolar dos filhos. Por exemplo, a freqüência de interação entre ambos os pais e seus filhos veio a favorecer o desempenho das crianças em aritmética, leitura, escrita e na pontuação total do TDE. As pesquisas apontam que uma boa interação entre pais e filhos auxilia no estabelecimento de um relacionamento seguro da criança com os pais, podendo, também favorecer os relacionamentos interpessoais das crianças com seus pares e a formação de um autoconceito satisfatório, aspectos que são maximizadores do desempenho acadêmico (Bolsoni-Silva \& Marturano, 2002; Cia \& Barham, 2005; Gomide, 2003; Marturano, 2004). Outro aspecto favorecedor do desenvolvimento social das crianças que veio a contribuir para o desempenho delas em leitura, escrita e na pontuação total do TDE foi o incentivo que ambos os pais dão aos seus filhos para que tenham contato com outras crianças.

Ainda em relação à participação de ambos os pais nas atividades escolares, culturais e de lazer dos filhos, pode-se verificar que alguns comportamentos relacionados às atividades escolares - como acompanhar o progresso escolar do filho, valorizar suas conquistas acadêmicas e auxiliá-lo nas lições de casa - estavam correlacionados com o desempenho das crianças em leitura, escrita ou total do TDE . Sendo assim, há indicativos de que a realização conjunta de algumas atividades acadêmicas das crianças com seus pais é crucial para o bom desempenho acadêmico dos filhos. È interessante mencionar a relação entre auxiliar os filhos nas lições de casa e o desempenho acadêmico dos filhos. Nesse estudo, não se questionou como seria esse auxílio dos pais: supervisionar o estudo, esclarecer dúvida, corrigir a tarefa, solicitar que o filho fale o que entendeu da matéria estudada ou realizar a tarefa para o filho, entre outros. Para que os filhos sintam confiança em sua capacidade acadêmica, os pais deveriam supervisionar as tarefas de casa e ajudar os filhos apenas quando solicitados. Essa ajuda deveria fazer com que os filhos raciocinassem para aprender a fazer sozinhos, e nunca que os pais fizessem as atividades para eles (Capelari, 2002; Cervera \& Alcázar, 2002).

Além disso, alguns comportamentos maternos de cuidados com os filhos (como auxiliar o filho nas atividades de higiene e pedir ao filho que organize os 
objetos pessoais) e alguns comportamentos paternos relacionados a atividades de lazer (passear e brincar com o filho) estavam positivamente correlacionados com o desempenho acadêmico das crianças em leitura, escrita, aritmética e na pontuação total do TDE. Essa correlação vem a demonstrar que, apesar de pais e mães interagirem com os filhos de maneiras e em atividades diferentes, ambos contribuem para alguns aspectos do desenvolvimento infantil.

Quanto à pontuação das crianças em aritmética, pôde-se verificar que estava positivamente correlacionada com o envolvimento do pai com seu filho, no que diz respeito à comunicação entre filho e pai e nas atividades de lazer que o pai realiza com seu filho. Tais resultados apontam para a necessidade de verificar quais os comportamentos paternos e as implicações desses comportamentos com o desenvolvimento infantil que poderiam auxiliar no desenvolvimento do raciocínio das crianças de forma a favorecer seu desempenho em aritmética.

Além disso, a relação entre o envolvimento paterno e o desempenho em aritmética das crianças se referia à comunicação filho-pai e às atividades de brincar e passear com os filhos. Para Piaget, os três fatores que explicam o desenvolvimento seriam a maturação, a experiência com os objetos e a experiência com as pessoas. O brincar/passear com os filhos permite que os pais estimulem diversos tipos de operações descritos por Piaget como essenciais para a construção do pensamento operacional concreto (classificação, seriação, conservação numérica, mediação, assim como a noção de reversibilidade, descentração e conservação), o que, por sua vez, torna-se a base para as operações lógico-matemáticas (Coll, Palacios \& Marchesi, 2004).

\section{CONSIDERAÇÕES FINAIS}

Este estudo levantou questões acerca do envolvimento parental que podem influenciar o desempenho acadêmico dos filhos. Pôde-se verificar, de modo geral, que quanto maior a freqüência de interação entre pais e filhos e da participação dos pais nas atividades escolares, culturais e de lazer dos filhos, maior o desempenho acadêmico das crianças. Infere-se que programas educacionais com mães/pais que tenham indicativo de pouco envolvimento ou envolvimento inadequado com os filhos poderiam aprimorá-lo e por conseqüência, resultar em um desenvolvimento mais saudável das crianças, como verificado em programas desenvolvidos em outros países (Bolsoni-Silva, Del Prette \& Del Prette, 2000;
Mahoney, Lord \& Carryl, 2005; Pinheiro, Haase, Del Prette, Amarante \& Del Prette, 2005).

Embora este estudo tenha sido conduzido com uma amostra de crianças restrita a apenas uma escola, os resultados confirmam alguns dados da literatura e sugerem pesquisas com amostras ampliadas, considerando diferentes estratos sociais. Deve-se ressaltar que a natureza dos dados deste estudo foi correlacional, e claras conclusões sobre a direção causal não podem ser estabelecidas (Thompson, Diamond, McWilliam, Snyder \& Snyder, 2005). Soma-se a isso o fato que os dados deste estudo baseiam-se no relato de crianças, podendo haver diferenças entre a percepção destas e o que realmente ocorre em seu dia-a-dia. Sendo assim, estudos longitudinais e observacionais seriam indicados para monitorar a influência do envolvimento parental sobre o desempenho acadêmico dos filhos ao longo do desenvolvimento infantil.

\section{REFERÊNCIAS}

Atzaba-Poria, N., Pike, A. \& Deater-Deckard, K. D. (2004). Do risk factors for problem bahavior act in a cumulative manner? An examination of ethnic minority and majority children through an ecological perspective. Jounal of Child Psychology and Psychiatry, 45(4), 707-718.

Ackerman, B. P., Brown, E. D. \& Izard, C. E. (2004). The relations between contextual risk, earned income, and the school adjustment of children from economically disadvantaged families. Developmental Psychology, 40(2), 204-216.

Brancalhone, P. G., Fogo, J. C. \& Williams, L. C. A. (2004). Crianças expostas à violência conjugal: avaliação do desempenho acadêmico. Psicologia: Teoria e Pesquisa, 20(2), 113-117.

Brasil, Ministério da Educação. (2002). Classe especial: ressignificando sua prática. Brasília: Secretaria de Educação Especial.

Bertolini, L. B. A. (2002). Funções paternas, maternas e conjugais na sociedade ocidental. Em A. L. B. Bertolini (Org.), Relações entre o trabalho da mulher e a dinâmica familiar (pp. 27-31). São Paulo: Vetor.

Bolsoni-Silva, A. T., Del Prette, A. \& Del Prette, Z. A. P. (2000). Relacionamento pais-filhos: um programa de desenvolvimento interpessoal em grupo. Psicologia Escolar e Educacional, 3(3), 203-215.

Bolsoni-Silva, A. T. \& Marturano, E. M. (2002). Práticas educativas e problemas de comportamento: uma análise à luz das habilidades sociais. Estudos de Psicologia, 7(2), 227-235.

Caballo, V. E. \& Simón, M. A. (2005). Manual de psicologia clínica e do adolescente: transtornos específicos. São Paulo: Santos.

Cabrera, N. J., Tamis-LeMonda, C., Bradley, R. H., Hofferth, S. \& Lamb, M. E. (2000). Fatherhood in the twenty first century. Child Development, 71(1), 127-136. 
Capelari, A. (2002). Modelagem do comportamento de estudar. Em M. Delitti (Org.), Sobre comportamento e cognição (pp. 3033). Santo André: Esetec.

Cervera, J. M. \&Alcázar, J. A. (2002). As relações pais-colégios. São Paulo: Quadrante.

Cia, F. \& Barham, E. J. (2005). A relação entre o turno de trabalho do pai e o autoconceito do filho. Psico, 1(1), 29-35.

Cia, F., D’Affonseca, S. M. \& Barham, E. J. (2004). A relação entre envolvimento paterno e desempenho acadêmico dos filhos. Paidéia: Cadernos de Psicologia e Educação, 14(29), 277-286.

Cia, F., Pereira, C. S., Del Prette, Z. A. P. \& Del Prette, A. (2006). Habilidades sociais parentais e o relacionamento entre pais e filhos. Psicologia em Estudo, 11(1), 73-81.

Cia, F., Williams, L. \& Aiello, A. L. R. (2005). Influências paternas no desenvolvimento infantil: revisão de literatura. Revista Psicologia Escolar e Educacional, 9(2), 225-233.

Cinamonm, G. R. \& Rich, Y. (2002). Gender differences in the importance of work and family roles: Implications for workfamily conflict. Sex Roles, 47(11/12), 531-541.

Coll, C., Palacios, J. \& Marchesi, A. (2004). Desenvolvimento psicológico e educação: psicologia evolutiva. Porto Alegre: ARTMED.

Cozby, P. C. (2002). Métodos de pesquisa em ciências do comportamento. São Paulo: Atlas.

Critério Brasil (2006). Associação brasileira de empresas de pesquisa. Recuperado em 05 de fevereiro de 2007, de $<$ http://www.abep.org.>.

D’Avila-Bacarji, K. M. G., Marturano, E. M. \& Elias, L. C. S. (2005). Suporte parental: um estudo sobre crianças com queixas escolares. Psicologia em Estudo, 10(1), 110-115.

Del Prette, Z. A. P. \& Del Prette, A. (2005). Psicologia das habilidades sociais na infância: teoria e prática. Petrópolis: Vozes.

Dessen, M. A. \& Costa, A. L. (2005). A ciência do desenvolvimento humano. Porto Alegre: ARTMED.

Duch, H. (2005). Redefining parent involvement in Head Start: A two-generation approach. Early Child Development and Care, 175(1), 23-35.

Englund, M. M., Luckner, A. E., Whaley, G. J. L. \& Egeland, B. (2004). Children's achievement in early elementary school: Longitudinal effects of parental involvement, expectations, and quality of assistence. Jounal of Educacional Psychology, 96(4), 723-730.

Ferreira, M. C. T. \& Marturano, E. M. (2002). Ambiente familiar e os problemas do comportamento apresentados por crianças com baixo desempenho escolar. Psicologia: Reflexão e Crítica, $15(1), 35-44$.

Flouri, E. (2004). Correlates of parents' involvement with their adolescent children in restructured and biological two-parent families: The role of child characteristics. International Journal of Behavioral Development, 28(2), 148-156.

Flouri, E. \& Buchanan, A. (2003). The role of father involvement in children's later mental health. Journal of Adolescence, 26, 6378.

Gargiulo, R. M. (2003). Special Education in contemporary society: An introduction to exceptionality. California: Wadsworth; Thommson Learning.
Gomide, P. I. C. (2003). Estilos parentais e comportamento antisocial. Em A. Del Prette \& Z. A. P. Del Prette (Orgs.), Habilidades sociais, desenvolvimento e aprendizagem (pp. 2160). Campinas: Alínea.

Hallahan, D. P. \& Kauffman, J. M. (2005). Exceptional learners: Introduction to special education. Boston: Allyn \& Bacon.

Hill, N. E, Castellino, D. R., Lansford, J. E., Nowlin, P., Dodge, K. A., Bates, J. E. \& Pettit, G. S. (2004). Parent academic involvement as related to school behavior, achievement and aspirations: Demographic variations across adolescence. Child Development, 75(5), 1491-1509.

Hong, S. \& Ho, H. (2005). Direct and indirect longitudinal effects of parental involvement on student achievement: SecondOrder latent growth modeling across ethnic groups. Journal of Educational Psychology, 97(1), 32-42.

Lamb, M. E. (1997). Fathers and child development: An introductory overview and guide. Em M. E. Lamb (Ed.), The role of the father in child developmental (pp. 1-18). New York: John Wiley \& Sons.

Mahoney, J. L., Lord, H. \& Carryl, E. (2005). An ecological analysis of after-school program participation and the development of academic performance and motivacional attributs for desadvantaged children. Child Development, 76(4), 811-825.

Marturano, E. M. (2004). Fatores de risco e proteção no desenvolvimento sócio-emocional de crianças com dificuldades de aprendizagem. Em E. G. Mendes, M. A. Almeida \& L. C. A. Williams (Orgs.), Avanços recentes em educação especial (pp. 159-165). São Carlos: EDUFSCar.

McDowell, D. J. \& Parke, R. D. (2005). Parental control and affect as predictors of children's display rule use and social competence with peers. Social Development, 14(3), 440-457.

Oliveira, E. A., Marin, A. H., Pires, F. B., Frizzo, G. B., Ravanello, T. \& Rossato, C. (2002). Estilos parentais autoritário e democrático-recíproco intergeracionais, conflito conjugal e comportamento de externalização e internalização. Psicologia: Reflexão e Crítica, 15(1), 1-11.

Overstreet, S., Devine, J., Bevans, K. \& Efreom, Y. (2005). Predicting parental involvement in children's schooling within an economically disadvantaged African American sample. Psychology in the Schools, 42(1), 101-111.

Ozgun, O. \& Honig, A. S. (2005). Parental involvement and spousal satisfaction with division of early childcare in Turkish families with normal children and children with special needs. Early Child Development and Care, 175(3), 259-270.

Pamplin, R. (2005). A interface família-escola na inclusão da criança com necessidades educacionais especiais. Dissertação de Mestrado Não-Publicada, Programa de Pós-Graduação em Educação Especial, Universidade Federal de São Carlos, São Carlos.

Pinheiro, M. I. S., Haase, V. G., Del Prette, A., Amarante, C. L. D. \& Del Prette, Z. A. P. (no prelo). Treinamento de habilidades sociais educativas para pais de crianças com problemas de comportamento. Psicologia: Reflexão e Crítica.

Ross, H., Stein, N., Trabasso, T., Woody, E. \& Ross, M. (2005). The quality of family relationships within and across generations: A social relations analysis. International Journal of Behavioral Development, 29(2), 110-119. 
Scaramella, L. V. \& Conger, R. D. (2004). Intergenerational continuity of hostile parenting and its consequences: The moderating influence of children's negative emoctional reactivity. Social Development, 12(3), 420-439.

Stein, L. M. (1994). Teste de desempenho escolar. São Paulo: Casa do Psicólogo.

Thompson, B., Diamond, K. E., McWilliam, R., Snyder, P. \& Snyder, S. W. (2005). Evaluating the quality of evidence from correlational research for evidence-based practice. Exceptional Children, 71(2), 181-194.

Wagner, A., Predebon, J., Mosmann, C. \& Verza, F. (2005).Compartilhar tarefas? Papéis e funções de pais e mães na família contemporânea. Psicologia: Teoria e Pesquisa, 21(2), 181-186.

Weber, L. N. D., Prado, P. M., Viezzer, A. P. \& Brandenburg, O. J. (2004). Identificação de estilos parentais: o ponto de vista dos pais e dos filhos. Psicologia: Reflexão e Crítica, 17(3), 323331.

Recebido em 24/08/2006 Aceito em 17/01/2007

Endereço para correspondência:

Fabiana Cia. Universidade Federal de São Carlos, Centro de Educação e Ciências Humanas, Departamento de Psicologia, Laboratório de Análise e Prevenção de Violência (LAPREV). Rodovia Washington Luís, Km 235, CEP 13565-905, São Carlos-SP. E-mail: fabianacia@hotmail.com 